\title{
Atención a la dependencia y cuidados sanitarios: obligados a entenderse*
}

\author{
Raúl del Pozo Rubio \\ Universidad de Castilla-La Mancha \\ Marta Ortega Ortega \\ Universidad Complutense de Madrid
}

\begin{abstract}
Resumen
Los sistemas sanitarios y de cuidados de larga duración (CLD) conforman dos pilares esenciales del estado de bienestar en España. Si bien el sistema de CLD nació como independiente del sanitario, su carácter y objetivos implican su necesaria integración, o al menos, coordinación con el sistema sanitario. La estrategia de atención integrada al paciente podría permitir un ahorro de recursos, realizar una óptima asignación de estos, ahorrar tiempos de espera y mejorar la calidad de vida de las personas con dependencia. En este trabajo se contextualiza la situación actual y se propone un conjunto de políticas públicas para el avance del estado de bienestar.
\end{abstract}

Palabras clave: sistema sanitario, sistema de cuidados de larga duración, cronicidad, integración, dependencia.

Clasificación JEL: E62, I10, I38.

\begin{abstract}
Both, the health care system and the long-term care system (LTC) are important pillars of the Spanish welfare state. Although Spanish LTC system was born independent of the health care system, its own nature and objectives suppose a necessary integration, or at least, coordination between both. This strategy would save resources, allocate them optimally, reduce waiting times and improve the quality of life of people with long-term care needs. In this chapter the current situation is contextualized and a set of public policies are proposed to achieve progress in the welfare state.
\end{abstract}

Keywords: health system, long-term care system, chronicity, integration, long-term care.

JEL classification: E62, I10, I38.

\section{Evolución histórica del estado de bienestar}

La segunda mitad del siglo xx, en concreto, los Treinta Gloriosos (1945-1975) conforman una etapa susceptible de ser considerada como un periodo dorado en la historia de los países occidentales (Esping-Andersen y Palier, 2010) en cuanto al

* Los autores desean agradecer la financiación obtenida del Proyecto ECO2017-83771-C3-1-R («Más allá de las fronteras económicas de las políticas de salud») del Ministerio de Ciencia, Innovación y Universidades (antiguo Ministerio de Economía y Competitividad). Programa Estatal de I+D+i orientada a los Retos de la Sociedad 2017. 
elevado y sostenido crecimiento económico, superior al de cualquier otro periodo anterior de la historia (Aldcroft, 1997). Si bien es cierto que los inicios de siglo estuvieron marcados por las dos guerras mundiales y la gran depresión, entre 1920 y 1940 se establecieron los cimientos institucionales del estado de bienestar (Ashford y Gimeno, 1989), consolidándose en la etapa de posguerra el crecimiento de la producción, de la economía y del sector público (Castells-Oliveres y BoschRoca, 1998).

En el caso de España, adicionando el padecimiento de una guerra civil y un periodo prolongado de dictadura, se obtiene como resultado un despegue tardío tanto en la economía nacional como en el gasto público respecto al resto de países occidentales (Tedde de Lorca, 1996), además de una notable desigualdad de producción y renta nacional en el territorio español; el gasto público comienza a crecer de manera relevante, y lo hace exponencialmente, pero a partir de 1974 (González-Páramo y Raymond Bara, 1988).

El fuerte crecimiento del gasto público experimentado durante esta etapa, sin precedentes históricos, ha dado lugar a diversas teorías que intentan argumentar y justificar los porqués de este crecimiento. González-Páramo y Raymond Bara (1988) y Albi et al. (2017) sintetizan estas teorías entre las que incluyen a la denominada Ley de Wagner (Musgrave, 1969; Bird, 1971). En esencia, el axioma sobre el que se sustenta esta ley se refiere a que la migración de la sociedad tradicional hacia una sociedad más industrializada y compleja, impulsa un efecto sustitución de cierta actividad privada por pública, con ocasión de asumir una serie de responsabilidades y servicios (transferencias de renta vía prestaciones) que el mercado ofrece de manera ineficiente o inequitativa y que solo se pueden corregir de manera colectiva (González-Páramo y Raymond Bara, 1988; Albi et al., 2017). Su inmediata repercusión en el caso de las economías avanzadas se refiere a que el Estado supera su rol tradicional, proveyendo no solo determinados bienes públicos como educación o sanidad a costes inferiores (a veces muy inferiores e incluso gratuitos) respecto del valor de mercado, sino también mediante transferencias de renta (pensiones, prestaciones por desempleo, etc.), que introduzcan los objetivos de igualdad social y justicia social en las instituciones (Ashford y Gimeno, 1989), y permitan configurar un nivel mínimo de dignidad del individuo (Albi et al., 2017), esto es, ocuparse de las «fuerzas sociales» (Luhmann y Vallespín, 1993), según esta teoría. En el caso de España, el tránsito del régimen dictatorial a la democracia en 1978 actuó como catalizador de trascendentes cambios sociales y de asunción de cobertura de importantes necesidades sociales por parte del Estado como antes nunca se había hecho. En este caso, un estado del bienestar descentralizado (Fusi y Palafox, 1997).

El corolario derivado de la asunción de estos servicios instiga un aumento de las necesidades de gasto público debido al desarrollo imprescindible de nuevas funciones diseñadas para satisfacer la demanda latente y nuevas necesidades (Luhmann y Vallespín, 1993; Jaén-García, 2004).

La naturaleza en la composición del gasto público y la distribución de participación en el mismo del ámbito público y privado depende de las circunstancias 
económicas, sociales, culturales e históricas de cada país (López Casasnovas, 2009), respondiendo a las necesidades sociales de cada época (Esping-Andersen y Palier, 2010). Mientras que los países de rentas bajas centran sus servicios básicos en el abastecimiento suficiente de agua potable, red de alcantarillado, etc., los países de rentas altas orientan su gasto social a otros servicios, tales como educación, sanidad o protección social. Aunque es preciso tener en cuenta que no solo difiere el empleo de recursos públicos, sino también el presupuesto de los mismos, reflejado en la existencia de una notable brecha económica (Alonso, 2009; Pérez-García, 2011), e incluso digital (Alonso, 2009) entre países de rentas altas, medias y bajas.

En España, el gasto público fundamental es aplicado al trinomio compuesto por educación, sanidad y protección social, cuya configuración permite aproximarse al concepto de estado de bienestar, derivado del inicial «capitalismo de bienestar» (Esping-Andersen, 1990) y de la realización de la inclusión política (Luhmann y Vallespín, 1993). Si bien su nacimiento estaba vinculado a paliar el masivo desempleo dimanado de la Gran Depresión que aquejaba a las economías occidentales (Castells-Oliveres y Bosch-Roca, 1998), en la actualidad se incluyen aquellas «intervenciones dirigidas a mejorar el bienestar social y la calidad de vida de la población» (Navarro, 2000), es decir, «las medidas adoptadas por el Estado para proteger a los ciudadanos contra aquellos riesgos frente a los cuales el mercado no ofrece una cobertura ni eficiente ni equitativa» (Conde-Ruiz y Ocaña-Orbis, 2007), incluyendo transferencias sociales (pensiones), intervenciones normativas (salud e higiene laboral, ambiental...) e intervenciones públicas encaminadas a producir empleo.

El trinomio citado se caracteriza en España por la universalización del derecho en su acceso, así como la gratuidad de su uso, con los matices pertinentes. En este sentido, basándonos en los últimos datos disponibles por funciones, en el año 2016 el gasto público general en España supuso el 42,2 por 100 del Producto Interior Bruto, donde más de la mitad de dicho gasto fue destinado a protección social como son las pensiones y prestaciones por desempleo (39,9 por 100$)$, sanidad (14,3 por 100) y educación $(9,5$ por 100) (Eurostat, 2018). Junto a ello, la necesidad del diseño y puesta en marcha de un modelo de cobertura a las personas en situación de dependencia en España, hizo florecer el cuarto pilar del trinomio con la aprobación en 2007 de la denominada Ley de Dependencia, cuyo objetivo fue la provisión de servicios o ayuda económica a personas con discapacidad y en situación de dependencia.

El objetivo del presente trabajo consiste en analizar la actual interrelación intrínseca existente entre los pilares del bienestar sanitario y de dependencia, revelando la necesidad de su coordinación y/o integración y cómo este segundo pilar no es sino una prolongación del primero.

\section{El nexo de unión entre sanidad y dependencia: la cronicidad}

Uno de los principales logros demográficos del ser humano durante el siglo Xx ha sido el importantísimo incremento de la esperanza de vida, pasando en el caso de 
España de una esperanza de vida de 34,8 años en 1900 a 83,1 años en 2017 (Instituto Nacional de Estadística, 2018). Se trata de la segunda mayor de los países de la OCDE, después de Japón, y está tres puntos por encima de la media de los países de la OCDE-35, siendo esta de 80,6 en el año 2015 (OCDE, 2017).

En este sentido, el estrato de población española con edad superior a 65 años adquiere un rol fundamental en la actualidad al tener un peso demográfico del 18,4 por 100 esperándose un incremento hasta alcanzar el 34,6 por 100 en 2066 (Abellán García et al., 2017). En el ámbito de la UE-28, la tasa de dependencia de la población mayor (definido como la población con edad superior a 65 años en relación a la población con edad comprendida entre 15 y 64 años) se espera que se incremente del 27,8 por 100 al 50,1 por 100 durante el periodo mencionado anteriormente (Comisión Europea, 2015).

Con el aumento de la edad aparece de manera inherente el padecimiento de determinadas enfermedades, que si bien no tienen por qué resultar mortales en el corto plazo, generan en el ser humano una senda de erosión de su salud, continuada de cronicidad. A partir de los 60 años, las grandes cargas de la enfermedad sobrevienen debido a la pérdida de la visión, audición y movilidad, así como a las enfermedades crónicas como las cardiopatías, accidentes cerebrovasculares, enfermedades respiratorias, cáncer y demencia (Organización Mundial de la Salud, 2015). La cobertura de la salud universal es uno de los treinta objetivos del desarrollo sostenible de las economías establecidos por la Organización Mundial de la Salud hasta el 2030. Este objetivo concreto pretende que todos los individuos del planeta puedan acceder a un rango de servicios completos para satisfacer sus necesidades (servicios de prevención, de curación y cuidados paliativos), y además, que dichos servicios sean provistos con calidad y eviten la entrada de catastrofismo financiero de los hogares cuando tengan que ser financiados (Organización Mundial de la Salud, 2016).

El nuevo escenario dibujado por las enfermedades crónicas parte del conjunto de un cambio en el perfil de la demanda sociosanitaria de las personas de mayor edad en la sociedad. Antares Consulting (2010) lo sintetiza en el esquema presentado en la Figura 1, donde se muestran las causas que avalan la respuesta común que los países de rentas altas han tenido frente al reto del envejecimiento y a sus nuevas necesidades, entre ellas, dar cobertura a las enfermedades crónicas y sus características específicas.

En concreto, entre otros matices, se ha intentado que una vez que estas enfermedades hayan sido correctamente diagnosticadas, y su tratamiento se haya hecho efectivo, la disciplina sanitaria no termine de ver cumplida su misión hasta que se hayan prestado los cuidados paliativos necesarios, si estos hubieran sido requeridos. 


\section{FIGURA 1}

\section{ESQUEMA DE MODELO CAUSAL: CAMBIOS EN EL PERFIL DE LA DEMANDA SOCIOSANITARIA}

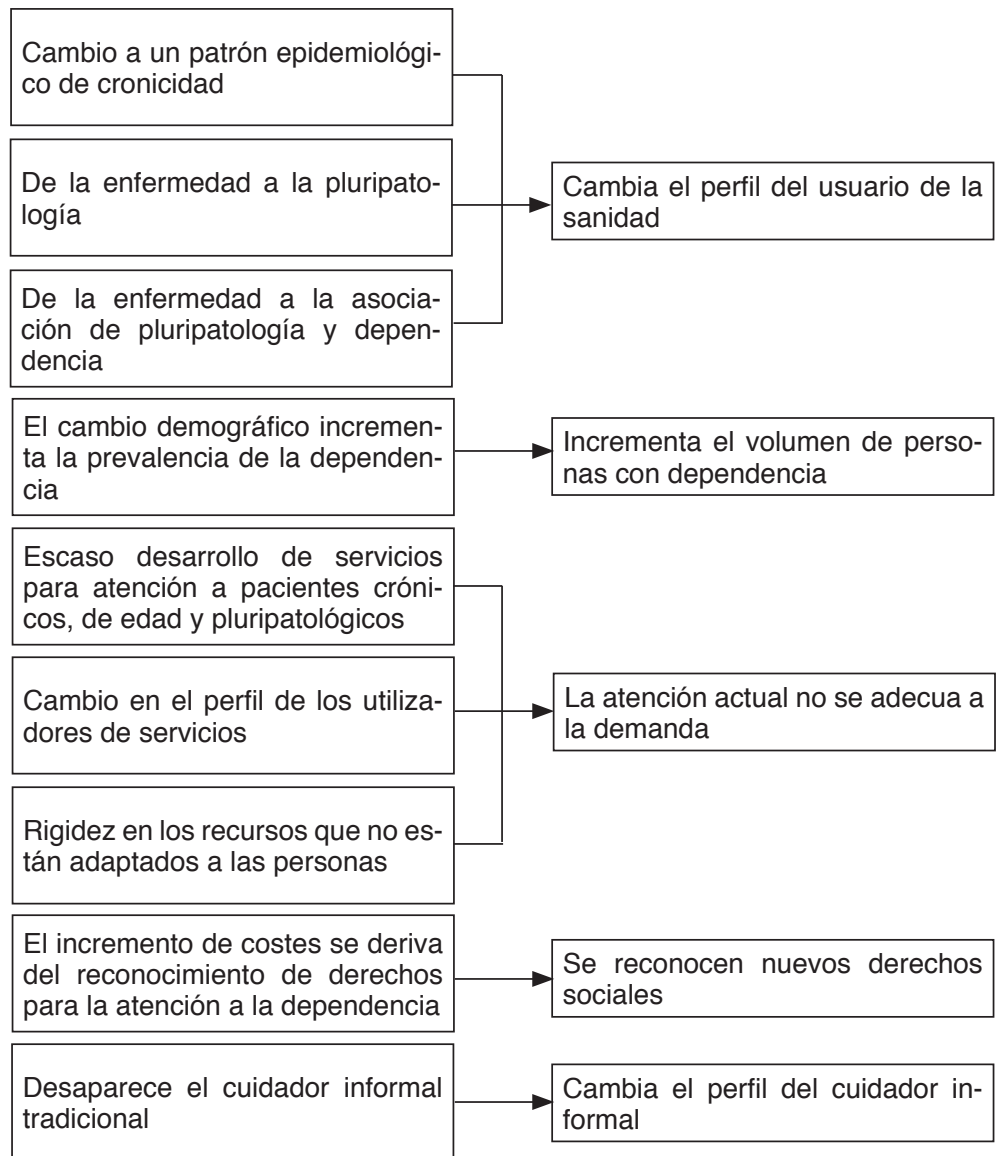

FUENTE: Antares Consulting (2010).

Una estrategia que se ha llevado a cabo con éxito en Reino Unido es la «estratificación» de pacientes crónicos basada en la Pirámide de Kaiser Permanente (Pines et al., 2015), consistente en segmentar a los pacientes en función de distintos niveles de necesidades de atención sanitaria con el fin de que el facultativo gestione mejor la enfermedad crónica logrando evitar asistencias a urgencias y/o ingresos hospitalarios innecesarios, mejorando de este modo la eficiencia del sistema (Osakidetza, 2010).

Como se observa en la Figura 2, los pacientes son estratificados según su nivel de cronicidad, observándose que los pacientes de alta complejidad requieren una gestión completa del caso y el 100 por 100 de cuidados profesionales, mientras que los pacientes crónicos en estadios iniciales de la enfermedad pueden autocuidarse y 


\section{FIGURA 2}

\section{PIRÁMIDE DE KAISER PERMANENTE AMPLIADA}

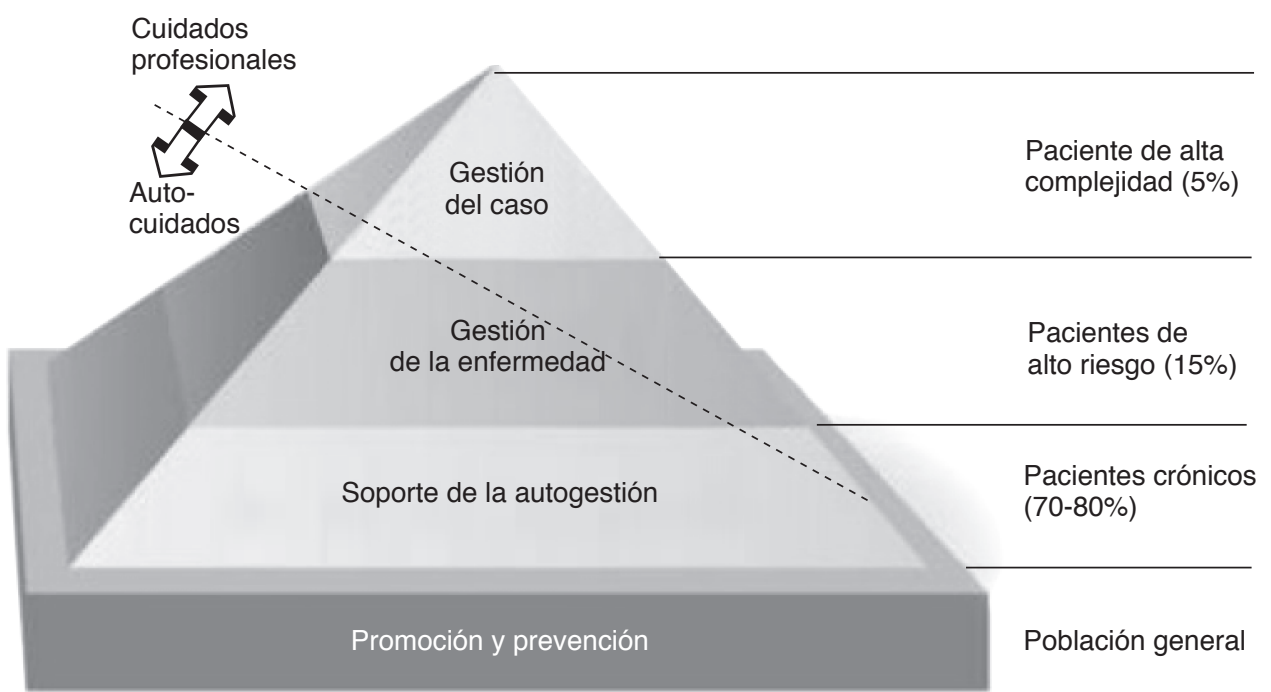

FUENTE: Osakidetza (2010).

es recomendable la ayuda y la orientación para la autogestión de la cronicidad. La principal meta de esta estrategia es la clasificación de los pacientes en el nivel correspondiente, y a partir de ahí, las implicaciones que se derivan de su situación para optimizar su atención.

En esta línea, Blay (2012) propone un modelo completo de atención integrado de cuidados para Cataluña en el que muestra la distribución de tipología de atención sanitaria (preventiva, curativa y paliativa) en función del tipo de morbilidad sufrida por los individuos, y cómo se reparte la atención al problema desde la óptica de gestión del problema vs. gestión del caso, representado en la Figura 3.

Este modelo se ha realizado a partir del análisis de la salud de la población catalana, y revela la distribución de la población según la situación de padecimiento de enfermedades crónicas: en este sentido, manifiesta que el 33 por 100 de la población no padece ninguna enfermedad, por lo que no precisa ningún tipo de cuidado (interviene la actividad sanitaria preventiva); un 61 por 100 padece algún tipo de comorbilidad no compleja, esto es, una o varias enfermedades de atención no compleja, donde intervienen los profesionales sanitarios en dos sentidos: por un lado intervención, y por otro, enseñanza a los pacientes de autogestión de tratamientos; un 2-3 por 100 de la población presenta algún tipo de morbilidad compleja, caracterizada por una fuerte intervención de profesionales sanitarios, igual que aquellos pacientes denominados complejos y enfermos avanzados, que representan un 1,5 por 100 de la población. Y por último, un 0,2 por 100 son enfermos terminales donde actúan los servicios paliativos en esencia (Blay, 2012). 


\section{FIGURA 3}

\section{MODELO DE ATENCIÓN INTEGRADO DE CUIDADOS}

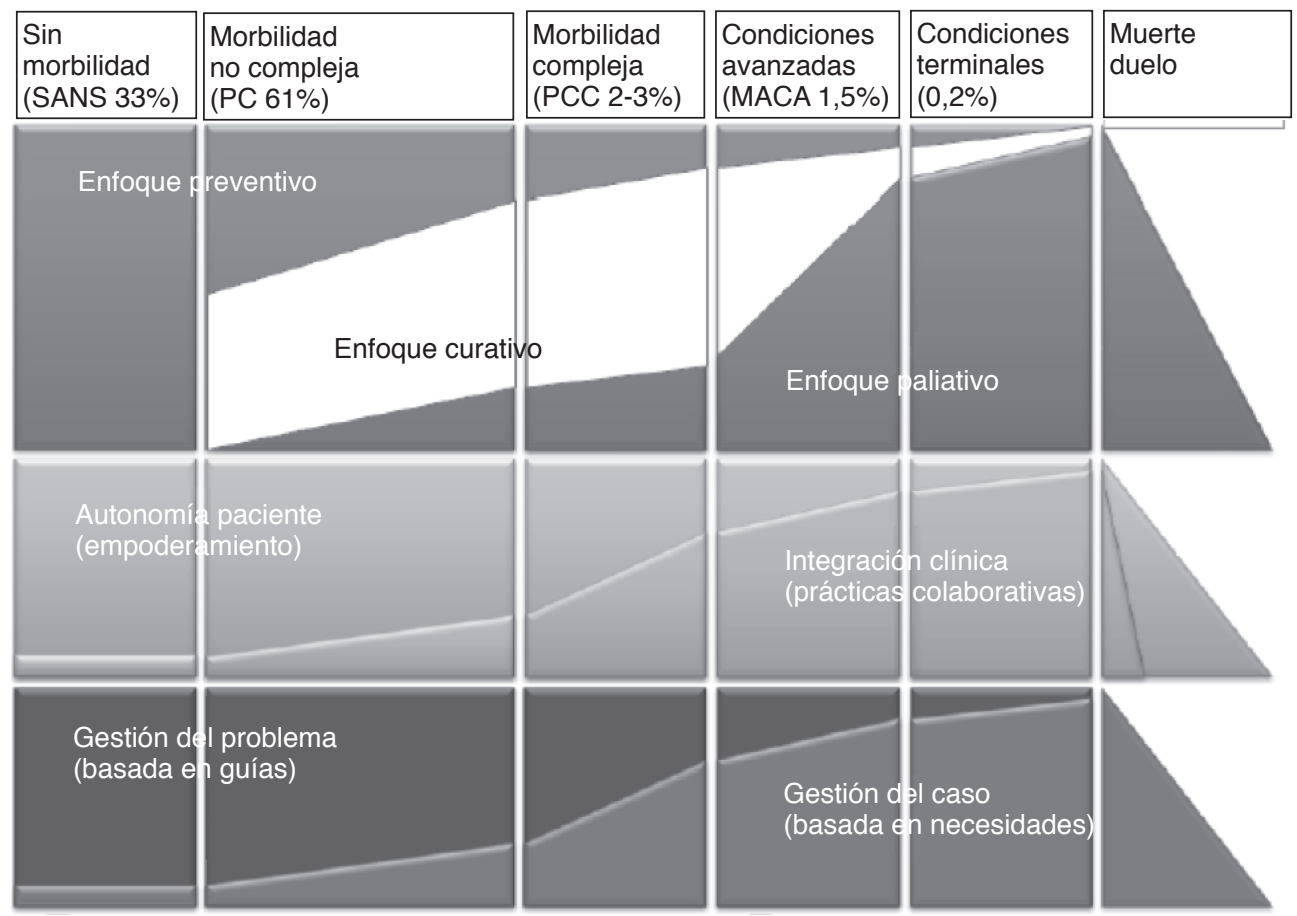

3 Puntos de inflexión en la biografía sociosanitaria

Transiciones paliativas

FUENTE: Blay (2012).

NOTA: SANS = Población sana; PC = Población con cronicidad (no compleja); PCC = Población con cronicidad compleja (comorbilidad compleja); MACA = Enfermos avanzados y población con cronicidad compleja avanzada.

Pese a la estratificación de pacientes en el sistema sanitario, en numerosas ocasiones las personas con enfermedades crónicas precisan una atención de cuidados que sobrepasa el alcance y la oferta de los servicios sanitarios. Fundamentalmente, se trata de la necesidad de apoyo para realizar tanto actividades básicas (aseo y cuidado personal, comer y beber, control de micción y defecación, vestirse, levantarse, acostarse, caminar, adquirir diversas posturas corporales, etc.) como actividades instrumentales de la vida diaria (ser capaz de usar adecuadamente el teléfono, preparación de las comidas, desempeño de actividades domésticas, realizar la compra, tomar la medicación, administración de las finanzas del hogar, etc.), precisando para su realización del inexorablemente apoyo de una tercera persona.

En este punto, aparece la necesidad de los cuidados de larga duración (CLD), definidos como el conjunto de prestaciones y servicios para atender las necesidades de las personas que no pueden realizar de manera autónoma las actividades de la vida diaria (Muir, 2017). Como respuesta a dicha necesidad y en plena decadencia de la 
anterior fase álgida del ciclo económico español se diseña y aprueba el cuarto pilar del estado de bienestar para España (López Casanovas y del Pozo, 2010), la Ley 39/2006, de 14 de diciembre, de Promoción de la Autonomía Personal y Atención a las personas en situación de dependencia, más conocida como la Ley de Dependencia. Esta Ley oferta un completo catálogo de prestaciones de servicios y económicas para atender dichas necesidades. Entre las prestaciones de servicios se recogen los servicios de atención residencial, centros de día/noche, ayuda a domicilio y teleasistencia. Por su lado, las prestaciones económicas se desagregan en prestación económica vinculada al servicio, prestación económica de asistencia personal y prestación económica para cuidados en el entorno familiar y apoyo a cuidadores no profesionales. Cabe señalar que esta última prestación se diseñó para formalizar la situación de aquellas personas (familiares o amigos) que proveían cuidados informales.

El desarrollo e implementación de la Ley de Dependencia supone la ampliación de derechos sociales cimentados sobre el reconocimiento formal de una atención que se había realizado tradicionalmente a través del cuidado en el hogar de manera altruista por la familia o amigos, denominado cuidado informal (García-Calvente et al., 2004; Antares Consulting, 2010) y que comienza a extinguirse como consecuencia de los nuevos cambios sociodemográficos en la sociedad española, tales como la incorporación de la mujer al mercado de trabajo, la reducción del tamaño y cambio en las estructuras familiares, aumento de las tasas de divorcio y reducción de las tasas de matrimonio, o los cambios en los patrones de migración rural-urbana e internacional (Mestheneos y Triantafillou, 2005; Antares Consulting, 2010).

Un breve análisis de los cuidados informales prestados en España permitirá situar al lector en contexto para comprender la carga familiar a la que nos referimos. En este sentido, nos focalizaremos en las personas que sufren discapacidad porque es uno de los colectivos en los que los cuidados se hacen más patentes e imprescindibles. Utilizamos la Encuesta sobre Discapacidades, Autonomía Personal y Situaciones de Dependencia (EDAD 08) (Instituto Nacional de Estadística, 2008) que ofrece información sobre discapacidades, deficiencias y limitaciones, además de información socioeconómica de la población española con discapacidad. Para cuantificar las horas de cuidados informales hemos clasificado previamente a las personas con discapacidad en los distintos grados de dependencia recogidos por la Ley (grados I, II y III), ya que no se dispone de dicha información directamente. Ello se ha realizado mediante la valoración del nivel de ayuda precisada para realizar las actividades básicas y las actividades instrumentales de la vida diaria recogidas en el baremo de valoración (Boletín Oficial del Estado, 2011) y en la encuesta utilizada, siguiendo una metodología similar a otros estudios (Sosvilla-Rivero y Moral-Arce, 2011; Oliva et al., 2011; del Pozo-Rubio et al., 2017). En concreto, la puntuación final que categoriza la discapacidad en los grados de dependencia I, II o III, es el resultado de la suma ponderada de cada actividad básica de la vida diaria en la que precisa de apoyo el individuo y el peso que cada actividad tiene en el cómputo global y por el nivel de apoyo que requiere cada individuo. Cabe señalar que la ponderación de cada actividad que recoge el propio baremo está intrínsecamente relacionada con la edad de la persona. 
En concreto, en la definición de la variable «grado de dependencia», la propia Ley de Dependencia transforma el criterio de morbilidad utilizado por Blay (2012) al número de veces que una persona necesita ayuda para realizar las actividades básicas de la vida diaria. En este sentido, si necesita ayuda al menos una vez al día o de manera intermitente, se dice que el grado de dependencia es moderado o grado I; si la persona precisa ayuda dos o tres veces al día, pero no de manera permanente, el grado de dependencia es II o dependencia severa; por último, si la persona precisa esa ayuda de manera permanente, se define como gran dependencia o grado III.

Se han calculado dos estimaciones del número de horas semanales de cuidado informal que precisa el individuo antes de la puesta en funcionamiento de la Ley, y por tanto, previamente a recibir algún tipo de prestación. La primera recoge los resultados puros de la encuesta, mientras que la segunda limita a 16 el número máximo de horas diario de cuidado informal que la persona con discapacidad puede recibir, al considerar que el cuidador informal debe de disponer al menos de ocho horas diarias para descanso (Ortega-Ortega et al., 2018; Peña-Longobardo y Oliva-Moreno, 2015).

Como muestra el Gráfico 1, la media de edad según grado de dependencia es de 62,3 años $($ d.e. 19,00$)$ para las personas con discapacidad pero sin dependencia (grado 0$) ; 70,74$ años $($ d.e. 18,00$)$ con grado I; 72,95 años $($ d.e. 18,70$)$ con grado II y para el grado III la media de edad es de 75,52 años (d.e. 20,03). Por tanto, se vislumbra cómo la dependencia concentra su severidad en edades superiores a los 65 años.

En esta misma línea, tal y como muestra el Gráfico 2, un 21,68 por 100 de las personas con discapacidad pero sin grado de dependencia recibe al menos una hora de cuidado informal; para las personas con grado I este porcentaje se incrementa hasta el 69,76 por 100; para las de grado II, hasta el 82,60 por 100; y para las de grado III, el 89,91 por 100 recibe, al menos, una hora diaria de cuidado informal.

Por tanto, como muestran los datos, el cuidado informal es muy relevante para la población con diversidad funcional. Sin embargo, este no es más que un ejemplo para comprender el coste de oportunidad que un familiar o amigo debiera de asumir para proporcionar la ayuda necesaria. Por ello, el reconocimiento formal de estas necesidades a través de la Ley de Dependencia, exhorta, a nuestro juicio, a un reconocimiento formal del Sistema de Dependencia dentro de la gestión del conjunto de servicios sanitarios y estratos de población atendidos por la actividad sanitaria. En otras palabras, la fusión íntegra y coordinada a nivel organizativo, de gestión y de información entre los distintos niveles del sistema de atención al enfermo (Figura 4).

En la Figura 4 proponemos el reconocimiento de las personas con dependencia, mediante la introducción formal de un nuevo sistema de cuidados y atenciones que están intrínsecamente relacionados con la edad de los individuos, con su nivel de salud y que se encuentra obligatoriamente inmerso entre los cuidados sanitarios y los CLD. Consideramos que las personas con dependencia precisan, al menos, de la coordinación entre servicios como condición suficiente y necesaria para que su atención ostente un prisma completo de sus necesidades, evitando sesgos de valoración y dilataciones en los tiempos de reconocimiento de necesidad y actuación. 


\section{GRÁFICO 1}

\section{HORAS DE CUIDADO INFORMAL SEMANALES PARA PERSONAS CON DISCAPACIDAD SEGÚN GRADO DE DEPENDENCIA Y EDAD MEDIA}

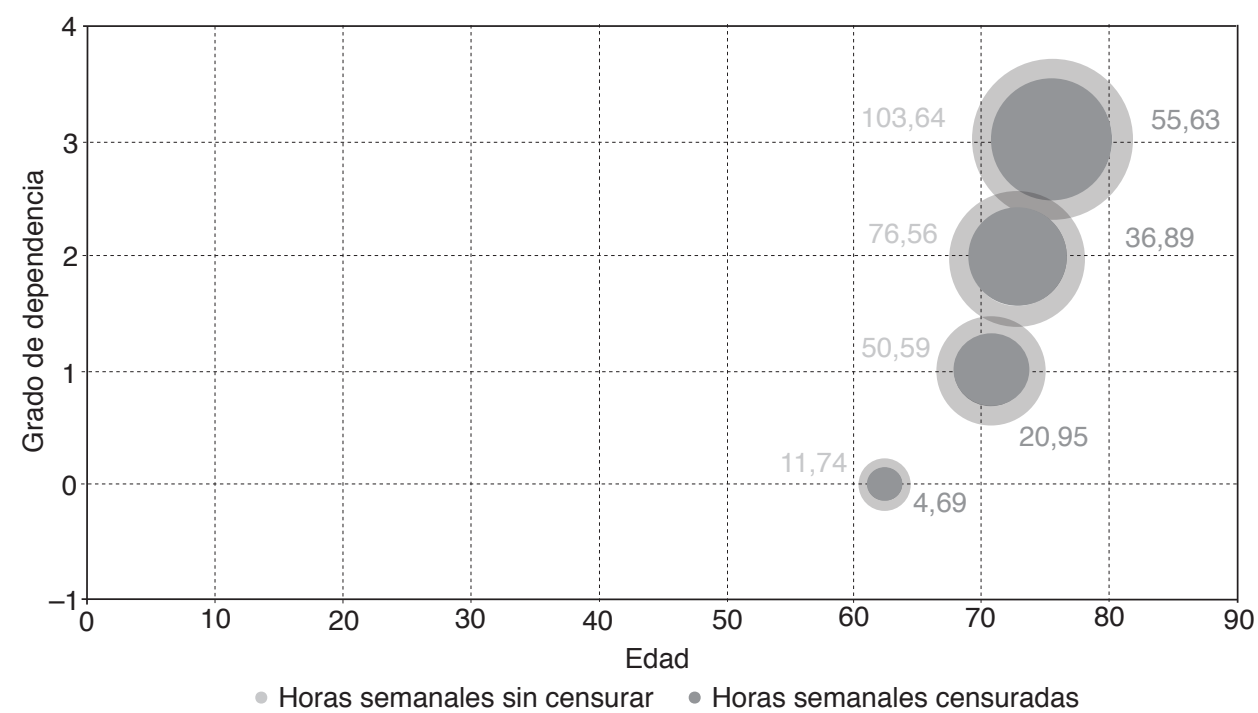

FUENTE: Elaboración propia a partir de EDAD 08.

\section{GRÁFICO 2}

PORCENTAJE DE EXISTENCIA DE CUIDADO INFORMAL Y HORAS DE CUIDADO INFORMAL SEMANALES PARA PERSONAS CON DISCAPACIDAD SEGÚN GRADO DE DEPENDENCIA

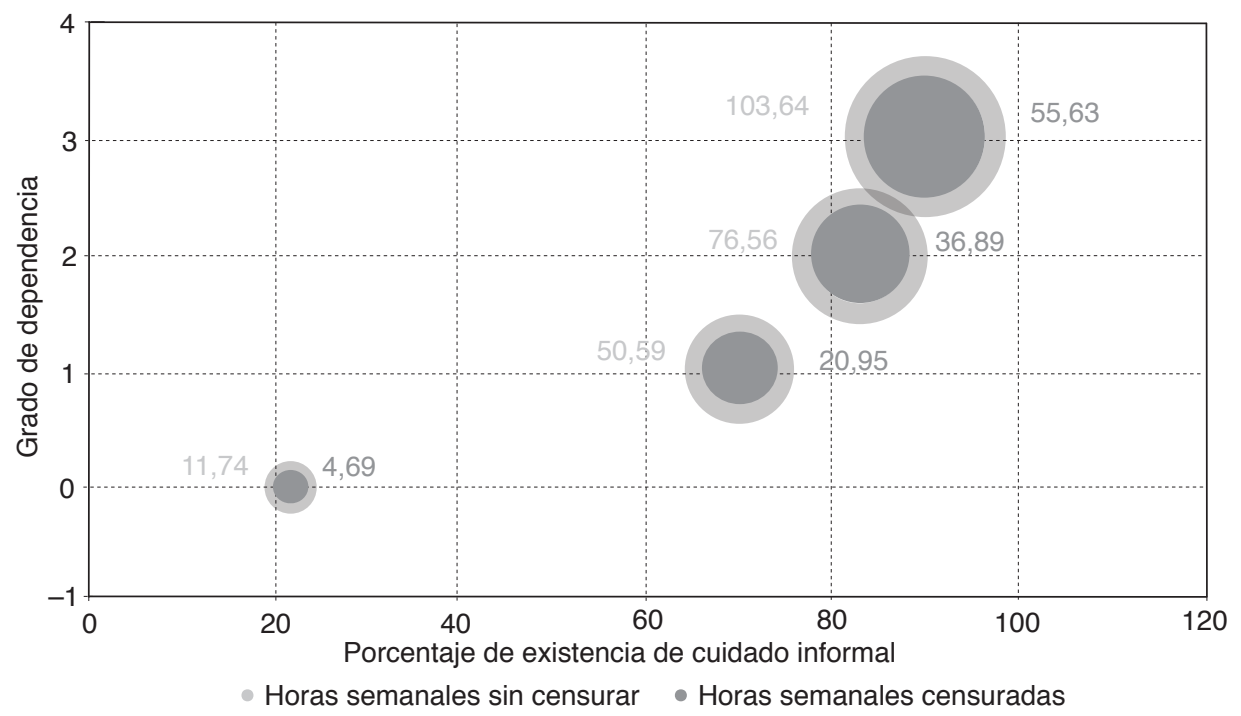

FUENTE: Elaboración propia a partir de EDAD 08. 


\section{FIGURA 4}

DISTRIBUCIÓN DE LOS CUIDADOS DE PREVENCIÓN, SANITARIOS, DE LARGA DURACIÓN Y PALIATIVOS SEGÚN ESTRATO DE POBLACIÓN

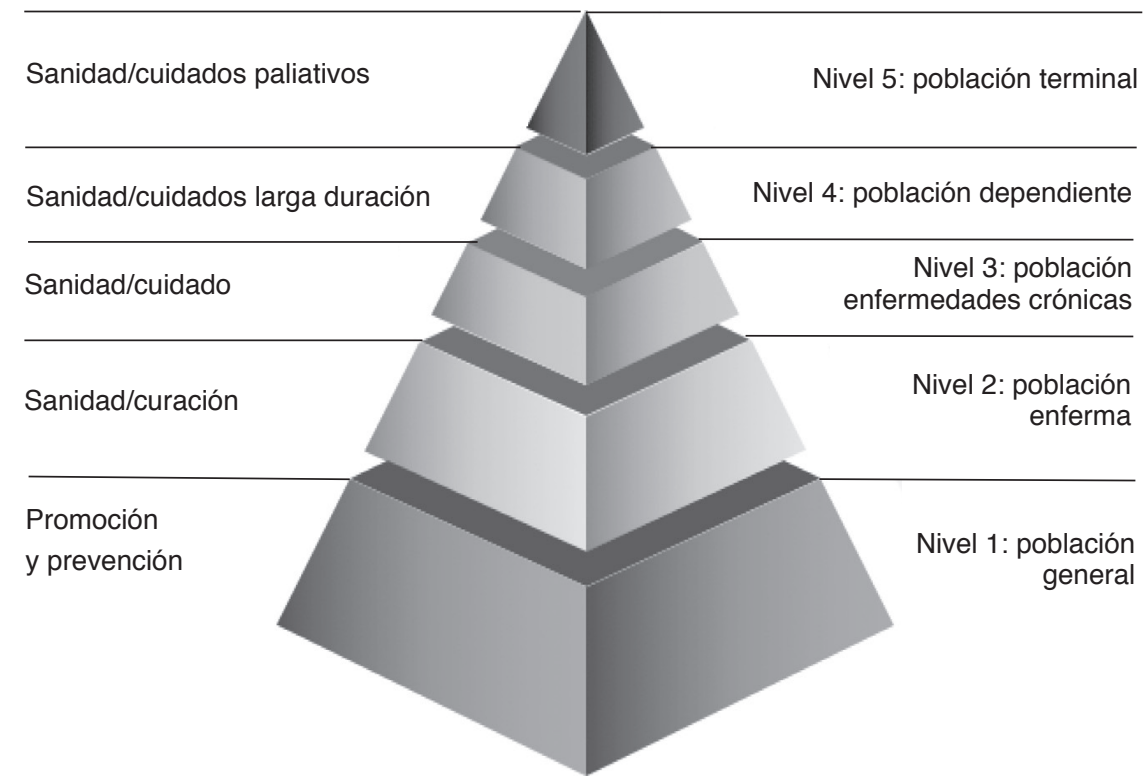

FUENTE: Elaboración propia a partir de la Pirámide Kaiser Permanente Ampliada.

\section{3. ¿Es necesaria la coordinación y/o integración entre sanidad y cuidados de larga duración?}

El reconocimiento formal de atención social a las personas dependientes supuso un adelanto en nuestra sociedad del bienestar. Desde su implementación, cabe destacar la asistencia formal a más de un millón de personas, pese a las limitaciones y deficiencias de la Ley en su puesta en funcionamiento, como las largas listas de espera entre la solicitud y la puesta en efectivo de la ayuda concedida. En abril de 2013 (último dato conocido) el tiempo medio de concesión de prestaciones era de 219 días, con importantes disparidades intercomunitarias (Tribunal de Cuentas, 2013).

Según la información ofrecida por el Servicio de Estadísticas de la Subdirección General Adjunta de Valoración, Calidad y Evaluación del SAAD-IMSERSO (IMSERSO, 2018), a fecha de 1 de enero de 2018, el sistema contaba con un total de 1.264.951 personas con derecho a recibir prestación de dependencia según la Ley; de éstas, el 32,74 por 100 tenían un grado I de dependencia, el 37,61 por 100 un grado II y el 29,65 por 100 restante un grado III. En la misma fecha, había 954.831 personas recibiendo efectivamente un total de 1.178 .011 prestaciones, lo que pone de manifiesto, por un lado, que de media cada persona recibe 1,24 prestaciones, y por otro lado, que más de 300.000 personas permanecen en lista de espera para recibir 
una prestación. En términos de prestaciones, el 46,83 por 100 de las concesiones son prestaciones de servicios (16,62 por 100 corresponden a servicio de atención residencial, 9,62 por 100 a centros de día/noche y 20,59 por 100 a servicios de ayuda a domicilio) y el 53,17 por 100 restante prestaciones económicas (11,70 por 100 de prestación económica vinculada al servicio, 0,70 por 100 de prestación económica de asistencia personal y 40,76 por 100 de prestación económica para cuidados en el entorno familiar y apoyo a cuidadores no profesionales). En relación a este último punto, no debemos olvidar que el legislador confirió inicialmente un claro carácter de preferencia a las prestaciones de servicios relegando a una situación de excepcionalidad las prestaciones económicas, en especial, a la prestación diseñada para el cuidado informal. Sin embargo, bien es cierto, como se observa, que una década después de la implementación de la Ley, cuatro de cada diez prestaciones de dependencia corresponden a la citada prestación económica para el cuidado informal.

Es importante destacar que de las personas que reciben prestación dos tercios son mujeres (65,5 por 100) mientras que el tercio restante son hombres; el 72,22 por 100 tiene una edad superior a 65 años, concentrándose más de la mitad de las mismas en el estrato de población de 80 años y superior (54,57 por 100). Esto muestra la acentuación del denominado envejecimiento del envejecimiento, cómo la población envejece más, y cómo el estrato de población más envejecido aglutina más problemas de salud y dependencia debido al propio hecho de envejecer.

Una de las características esenciales de las personas con dependencia es el tránsito continuado entre servicios sanitarios (atención primaria y especializada u hospitalaria) y los servicios sociales. Esto es, existe un importante segmento de población que comparte la necesidad de cuidados sanitarios y de cuidados de larga duración, entre otras cosas, debido a la avanzada edad de la población con dependencia y las comorbilidades existentes, como se ha comentado anteriormente. Sin embargo, cuando desde los servicios sociales se tiene que hacer uso de los sanitarios, en diversas ocasiones las necesidades apremian la atención en tiempo y forma: en este punto, se precisa información sociodemográfica para atender al individuo con la mayor garantía y calidad posibles. Por otro lado, cuando la dependencia es sobrevenida y los servicios de salud atienden a los pacientes, una vez que estos servicios han realizado su función, y su fase curativa y de control han sido llevadas a cabo, la siguiente fase se corresponde con la normalización e incorporación a la vida diaria, situación altamente complicada cuando las limitaciones asociadas a su enfermedad desarrollan una dependencia parcial o total en el individuo. Ello implica que los tiempos de trabajo y actuación sobre sus necesidades debieran de ser minimizados, por lo que una coordinación adecuada sobre su intervención sanitaria y atención de cuidados no solo es precisa, sino que de ella depende el éxito o el fracaso de las intervenciones en los pacientes y del sistema de bienestar.

Bengoa y Nuño (2008) desgranan detalladamente la relevancia de la conjunción de ambos términos «atención integrada» y «coordinación sociosanitaria», a partir de distintos modelos de atención analizados en la literatura. En primer lugar, el término atención integrada, pese a la falta aún de consenso en su interpretación, puede enten- 
derse como el «conjunto de técnicas y modelos organizativos designados para crear conectividad, alineación y colaboración entre la atención sanitaria y social (y dentro de ellas), en los niveles de financiación, gestión y provisión» (Kodner y Kyriacou, 2000). Se trataría en cualquier caso de integración de tres ámbitos: integración de la planificación, integración de la gestión e integración de la prestación efectiva de servicios (Oliva, 2014; Jiménez-Martín et al., 2011). En segundo lugar y de manera complementaria, la coordinación sociosanitaria es definida en España como «el conjunto de acciones encaminadas a ordenar los sistemas sanitario y social para ofrecer una respuesta integral a las necesidades de atención sociosanitaria que se presentan simultáneamente en las personas mayores dependientes» (INSALUD-IMSERSO, 1995). En otras palabras, la coordinación puede entenderse como la relación entre organizaciones independientes y la integración como la fusión de esas organizaciones (Bengoa y Nuño, 2008). Partiendo de esto, Bengoa y Nuño (2008) funden los conceptos de atención integrada y coordinación sociosanitaria, donde ponen de manifiesto que los recursos sociales y sanitarios tienen por objeto ser ordenados de acuerdo a cumplir el objetivo de atender las necesidades sociosanitarias (ampliando el concepto de enfermedades crónicas) de los individuos. Sin embargo, existen múltiples combinaciones entre coordinación e integración: completa integración vs. completa coordinación; integración de la planificación (gestión y provisión coordinadas); integración de la planificación y de la gestión (provisión coordinada); integración de la planificación, la gestión y la provisión (en este último caso, cuando los perfiles de atención a pacientes lo permitan, según las necesidades; en los restantes, obligatoriamente provisión coordinada).

Tratando de dar respuesta a estas necesidades sociosanitarias, son varias las autoridades que ya han apostado por un tipo de atención sociosanitaria integrada. Dentro de la evidencia empírica destaca el trabajo de Beland y Hollander (2011), en el que se realiza una revisión sistemática internacional de los modelos de integración sanitaria y CLD para personas mayores frágiles. Destacan la existencia de dos tipos de modelos básicos de atención sanitaria integrada: un modelo comunitario o local sustentado en la coordinación entre asistencia sanitaria y social, centrado en la asistencia domiciliaria; y un modelo a mayor escala caracterizado por tener una autoridad administrativa única, un único presupuesto en el que se incluyen tanto servicios sanitarios como sociales (residenciales o domiciliarios), y cuya autoridad podía ser desde local hasta nacional, obviamente con mayor dificultad de implementación. Independientemente del modelo adoptado por cada país, Beland y Hollander (2011) destacan como factor clave del éxito la necesidad de coordinación efectiva entre servicios de distinta naturaleza.

En nuestro contexto nacional se están realizando importantes avances regionales en torno a la atención integrada destacando como líderes de dicho cambio las Comunidades Autónomas de Madrid, Andalucía, Cataluña, Castilla y León o País Vasco (Ministerio de Sanidad, Servicios Sociales e Igualdad (2012); Consejería de Sanidad de la Comunidad de Madrid (2013); Direcció General de Regulació, Planificació i Recursos Sanitaris de la Generalitat de Catalunya (2012); Consejería de Salud de 
la Junta de Andalucía (2012); Consejería de Sanidad de la Junta de Castilla y León (2013); Departamento de Sanidad y Consumo del País Vasco (2010)). El Observatorio de Modelos Integrados de Salud (OMIS) se encarga de identificar y analizar experiencias de coordinación de los sectores social y sanitario en España y señala a la Comunidad Valenciana, Extremadura y Asturias como las comunidades más recientes en avanzar en esta línea a lo largo del año 2016. Según este Observatorio, la apuesta del resto de comunidades es poco apreciable (New Health Foundation, 2017). En este sentido, Andalucía se caracteriza por el desarrollo de un itinerario de atención compartida para personas con enfermedad grave crónica y sus familiares; la Comunidad de Madrid también se enmarca en la atención de pacientes con enfermedades crónicas; Castilla y León destaca por su impulso en pacientes crónicos pluripatológicos complejos y con enfermedades mentales; Cataluña ha implementado un modelo de atención integrada social y sanitaria fundada en la cronicidad con una gestión estable en la actualidad; y el País Vasco destaca entre el resto de regiones por sus estrategias de acción centradas en la ciudadanía, dando respuestas a las necesidades sociosanitarias individuales y colectivas (New Health Foundation, 2017). Sin embargo, la falta de consenso en los modelos de atención integrada entre regiones y su leve puesta en práctica, nos arrojan unos resultados débiles, fundamentalmente teóricos, dificultando la postulación de un modelo concreto a nivel nacional. Se trata de actuaciones más de forma que de fondo: si bien los diseños e intenciones son contundentes, los resultados obtenidos reflejan tímidos avances. En cualquier caso, el diseño de políticas sociosanitarias a partir del diseño de estrategias específicas de actuación desde los gobiernos estatal y comunitario no solo son procedentes, sino que su rapidez en diseño y formulación son precisadas por el segmento de población dependiente.

Tomaremos como ejemplo el caso de País Vasco, dado que su apuesta ha sido, junto con la catalana, la más visible, alcanzando un gran reconocimiento a nivel internacional según el OMIS. El País Vasco destaca por el encabezamiento pionero en políticas y estrategias de cronicidad (Departamento de Sanidad y Consumo del País Vasco, 2010). Como ejemplo, la elaboración de las cinco políticas y catorce proyectos estratégicos recogidos en el Cuadro 1 (Nuño-Solinís, 2016). Entre las conclusiones principales derivadas de la evaluación de la implementación de este paquete estratégico en plena crisis económica española, se han observado cambios importantes en la cultura de integración asistencial de gestores y clínicos, junto a una progresiva orientación de las organizaciones hacia el modelo de atención basado en la cronicidad. Destacan, como resultados relevantes, la reducción en utilización hospitalaria, readmisión de enfermos crónicos o la percepción de la coordinación social y sanitaria entre los pacientes. No obstante, resaltan que la resistencia o pasividad de grupos de interés importantes en el proceso de cambio fue una de las principales contrariedades halladas en su apuesta (desde el conjunto de decisores políticos hasta colectivos de trabajadores), lo que ha redundado en conseguir una integración con marcados tintes de debilidad (Urtaran-Laresgoiti et al., 2018; Nuño-Solinís, 2016). 


\section{CUADRO 1}

\section{POLÍTICAS Y PROYECTOS ESTRATÉGICOS DE LA ESTRATEGIA DE CRONICIDAD EN EL PAÍS VASCO}

\begin{tabular}{|c|c|c|c|c|}
\hline $\begin{array}{c}\text { Visión } \\
\text { poblacional }\end{array}$ & $\begin{array}{c}\text { Prevención } \\
\text { y promoción }\end{array}$ & $\begin{array}{l}\text { Autonomía } \\
\text { del paciente }\end{array}$ & $\begin{array}{l}\text { Continuo de } \\
\text { atención }\end{array}$ & $\begin{array}{l}\text { Intervenciones } \\
\text { personalizadas }\end{array}$ \\
\hline $\begin{array}{l}\text { 1. Estratificación } \\
\text { y targeting de la } \\
\text { población } \\
\text { 14. Innovación } \\
\text { desde los } \\
\text { profesionales } \\
\text { clínicos }\end{array}$ & $\begin{array}{l}\text { 2. Intervenciones } \\
\text { sobre los } \\
\text { principales } \\
\text { factores de riesgo }\end{array}$ & $\begin{array}{l}\text { 3. Autocuidado } \\
\text { y educación al } \\
\text { paciente: paciente } \\
\text { experto } \\
\text { 4. Apoyo a } \\
\text { la adopción } \\
\text { de las nuevas } \\
\text { tecnologías } \\
\text { por parte de las } \\
\text { asociaciones } \\
\text { de pacientes } \\
\text { crónicos }\end{array}$ & $\begin{array}{l}\text { 5. Historia clínica } \\
\text { unificada } \\
6 \text {. Atención } \\
\text { clínica integrada } \\
\text { 7. Desarrollo } \\
\text { de hospitales de } \\
\text { subagudos } \\
\text { 8. Competencias } \\
\text { avanzadas de } \\
\text { enfermería } \\
\text { 9. Colaboración } \\
\text { sociosanitaria } \\
\text { 10. Financiación } \\
\text { y contratación }\end{array}$ & $\begin{array}{l}\text { 11. OSAREAN: } \\
\text { centro multicanal } \\
\text { 12. Receta } \\
\text { electrónica } \\
\text { 13. Centro de } \\
\text { investigación de } \\
\text { cronicidad }\end{array}$ \\
\hline
\end{tabular}

FUENTE: Nuño-Solinís (2016).

Siguiendo esta última línea, Bengoa (2015) advierte de tres problemas relevantes que pueden frenar el proceso de transformación y adaptación del sector sociosanitario: en primer lugar, existe una visión individual y compartida por parte de muchos actores principales del sector sobre que un esquema de atención basado en gestión de agudos se configura como la mejor elección posible; en segundo lugar, una profunda modificación del sector hacia un nuevo objetivo es imposible si no se cuenta con la complicidad de los profesionales de la salud, puesto que se precisa inexorablemente aunar la excelencia clínica y la conducción de los procesos de gestión; por último, es necesario el liderazgo de esta transformación. En cualquier caso, el tránsito de los sistemas de salud hacia una optimización del cuidado a pacientes crónicos requiere cambios específicos en la práctica clínica diaria, educación y formación clínica, medidas de calidad, investigación clínica y diseño de políticas (Bierman y Tinetti, 2016).

Hay que señalar que pocos son los estudios que se han llevado a cabo para evaluar económicamente la atención integrada, y la comparativa entre los existentes es complicada debido a la falta de consenso en la definición y acotaciones de la «atención integrada» a nivel internacional (Desmedt et al., 2016; Nolte y Pitchman, 2014). Pese a ello, los últimos estudios muestran un beneficioso impacto económico de los modelos de atención integrada en los pacientes crónicos reflejando a su vez grandes beneficios en la salud (Cronin et al., 2017; Desmedt et al., 2016). Si bien es cierto que, en futuras investigaciones, la atención integrada, ya sea entendida como una intervención que como tal ha de ser coste-efectiva o como una estrategia de gestión innovadora que engloba múltiples niveles, ha de ser evaluada a largo plazo tanto en términos económicos como de resultados en salud, con el objetivo de generar conclusiones apropiadas para la correcta toma de decisiones (Nolte y Pitchman, 2014). 
Hasta el momento, la evidencia empírica nacional en el ámbito sanitario, ha vislumbrado que la integración entre servicios de atención primaria y hospitalaria es positiva, tal y como muestra la revisión sistemática llevada a cabo por Corral-Gudino et al. (2017), especialmente beneficiosa en los resultados observados con el autocuidado. Asimismo, un reciente trabajo de Costa-Font et al. (2016) muestra cómo el desarrollo de la Ley de Dependencia ha implicado una reducción significativa tanto en admisiones hospitalarias como en los días de estancia hospitalaria, especialmente en aquellas regiones que coordinan e integran sistemas de salud y bienestar social. En este sentido, un análisis del impacto económico podría complementar la información aportada para la toma de futuras decisiones políticas.

\section{Avances hacia el futuro}

La atención a la cronicidad se postula como el camino marcado por la agenda médica, donde los avances tecnológicos y clínicos pretenden lograr el control de la enfermedad evitando la mortalidad y mejorando la calidad de vida de los individuos. Cinco son las enfermedades crónicas más comunes en el mundo según la Organización Mundial de la Salud: el cáncer, las enfermedades cardiovasculares, mentales o neurológicas, respiratorias y las osteoarticulares (American Cancer Society, 2018; Organización Mundial de la Salud, 2015). Muchas de estas patologías, por el propio transcurso de la enfermedad, es probable que desemboquen en una situación de dependencia del individuo y de necesidad de ayuda para el desempeño de las actividades básicas o instrumentales de la vida diaria, por lo que la sociedad ha de ser corresponsable del desarrollo de sistemas que permitan el acogimiento y la gestión integral de la enfermedad y la dependencia en un marco de bienestar. La Ley de Dependencia es un camino iniciado hacia el reconocimiento formal de la situación de dependencia sobrevenida por las distintas enfermedades que la generan.

Sin embargo, dada la joven maduración de la Ley de Dependencia, son escasos los trabajos de necesidades de integración o coordinación de estructuras sanitaria y social con datos reales, así como de evaluación de las exiguas iniciativas llevadas a cabo. Los cambios sociales producidos en las últimas décadas como el aumento de la esperanza de vida, la cronicidad de las enfermedades ante la mortalidad o la inminente desaparición de los cuidadores informales, suponen una llamada de atención, como mínimo, a la coordinación de los sistemas de asistencia sanitaria y de asistencia social: esto es, la conexión del cuidado sanitario del enfermo con las ayudas sociales al mismo y su cuidador como punto de partida, y su trascendencia para el resto de ámbitos implicados. Por ello, ambos sistemas deben inexorablemente ejercer una función complementaria e ir de la mano, compartiendo información relativa a la gestión del paciente.

Se ha observado que el tránsito de una situación de no dependencia a dependencia moderada supone un incremento en el gasto que oscila entre el 150 por 100 y el 400 por 100, en función de la edad de la persona, siendo el gasto de atención social el que 
más claramente aumenta, entre un 500 por 100 y un 2.000 por 100 (Jiménez-Martín et al. 2011). En este sentido, si bien una de las características esenciales de la definición de dependencia es el «carácter permanente» de las limitaciones para realizar las actividades básicas de la vida diaria, el propio colectivo de trabajadores sociales que trabaja directamente con las personas dependientes ha observado, en un número importante de casos, que tras la puesta efectiva de la prestación, la situación sociosanitaria de la persona ha mejorado, observándose una reducción de sus limitaciones y su dependencia, y en consecuencia, una potencial reducción del grado de dependencia. Son necesarios por tanto estudios enfocados en esta dirección, puesto que además de poder permitir una mejor planificación de recursos públicos (y privados) a lo largo del ciclo vital de las personas, contribuyen a retrasar y reducir la duración de las situaciones más severas de dependencia, que por otro lado, son las que mayores cantidades de recursos sanitarios consumen. Por tanto, una atención conjunta de servicios sanitarios y sociales podría hacer posible el ahorro de costes y mejorar de la eficiencia del sistema (Jiménez-Martín et al., 2011).

Una propuesta muy discutida desde los inicios del diseño de la Ley se refiere a su financiación: si dicha Ley no debía haber sido sino una prolongación de las prestaciones recogidas por el catálogo de la Seguridad Social, y su financiación, la misma que la de esta. No parece lógico que un sistema de financiación separado y estanco de servicios sanitarios y sociales permita asignar de manera eficiente los recursos de ambos sistemas (Jiménez-Martín et al., 2011). Por tanto, un sistema conjunto que considere sanidad y bienestar social como subsistemas, y que cuente con una financiación conjunta e integrada recogida en la correspondiente Ley Orgánica de Financiación de las Comunidades Autónomas en lugar de dotación en los Presupuestos Generales del Estado, permitiría que la integración fuera, cuanto menos, exhortativa.

En este sentido, un debate sobre la financiación pública del nuevo marco de dependencia sería relevante, pudiéndose proponer la creación de nuevas herramientas fiscales dada la actual insuficiencia financiera mostrada, ya sea mediante el afianzamiento de los copagos introducidos en la reforma legislativa de 2012 según capacidad económica, o mediante el fomento fiscal de sistemas de aseguramiento público, privado o mixto de la dependencia.

Los autores se unen indudablemente a la propuesta de la creación de una única área de política denominada «sociosanitaria», que pudiera abarcar de manera integral la infraestructura de los servicios sanitarios y los servicios sociales y que permitiera reducir los tiempos de espera en cualquiera de los trámites burocráticos a realizar, no solo intervenciones sanitarias o recepción efectiva de prestaciones, sino también esperas de notificaciones, valoraciones, baremaciones o revisiones de grado y prestaciones. Tanto el personal que participa en todos los ámbitos inherentes al sistema sociosanitario como las herramientas informáticas que recogen la información de las personas con dependencia son figuras clave. Sin embargo, la culminación con éxito de la integración de los sistemas sanitario y social no está exenta de importantes limitaciones y elevados riesgos, fundamentalmente de contenido y de proceso. Es por este motivo que consideramos, como garante del éxito de la conjunción de las 
dos áreas citadas, un paso previo hacia una coordinación profunda y contundente de ambos sistemas. En primer lugar, avances en la coordinación de los ámbitos de planificación y gestión permitirían construir unos verdaderos y consolidados cimientos de la política sociosanitaria, donde una única autoridad se postularía como piedra angular de una posterior integración de actividad. Tras ello, dado que la integración de la prestación efectiva de la cartera de servicios solo se podría realizar con perfiles de atención y necesidad similares, y bajo criterios de idéntica naturaleza, una segregación de prestaciones que distinga entre las orientadas a la integración y las encaminadas a una coordinación máxima, permitiría consolidar el proceso de fusión de las dos áreas en una. Todo ello sin soslayar la figura de la persona dependiente como referencia.

En suma, se propone una perfecta coordinación entre los servicios sanitarios y sociales, como paso previo a una futura integración completa a largo plazo de los sistemas sanitario y social, en el que se repartan competencias y responsabilidades, comenzando con una estructura jerárquica de seguimiento del paciente, siguiendo por el tratamiento clínico de la enfermedad, continuando con el mantenimiento y control de la cronicidad a nivel sanitario, e incluyendo el eslabón de la asistencia de la dependencia previa a los cuidados paliativos.

\section{Referencias bibliográficas}

[1] ABELLÁN GARCÍA, A.; AYALA GARCÍA, A. y PUJOL RODRÍGUEZ, R. (2017). «Un perfil de las personas mayores en España, 2017. Indicadores estadísticos básicos». Informes de Envejemiento en Red, 15.

[2] ALBI, E.; GONZÁLEZ-PÁRAMO, J.; URBANOS, R. y ZUBIRI, I. (2017). Economía Pública I. Barcelona, Ariel, 4. . ed.

[3] ALDCROFT, D. H. (1997). Historia económica europea, 1914-1980. Barcelona, Crítica.

[4] ALONSO, J. A. (2009). Lecciones sobre economía mundial. Madrid, Thomson Civitas.

[5] AMERICAN CANCER SOCIETY (2018). «Managing cancer as a chronic illness». Disponible en www.cancer.org/treatment/survivorship-during-and-after-treatment/whencancer-doesnt-go-away.html

[6] ANTARES CONSULTING (2010). «Claves para la construcción del espacio sociosanitario en España». Disponible en www.antares-consulting.com.

[7] ASHFORD, D. E. y GIMENO, B. (1989). La aparición de los Estados de Bienestar: Centro de Publicaciones, Ministerio de Trabajo y Seguridad Social.

[8] BELAND, F. y HOLLANDER, M. J. (2011). «Integrated Models of Care Delivery for the Frail Elderly: International Perspectives». Gaceta Sanitaria, 25, 38-46.

[9] BENGOA RENTERÍA, R. (2015). «El reto de la cronicidad en España: mejor transformar que racionar». Gaceta Sanitaria, 29 (5), 323-325.

[10] BENGOA, R. y NUÑO, R. (2008). Curar y cuidar. Innovación en la calidad y la gestión de las enfermedades crónicas. Una guía práctica para avanzar.

[11] BIERMAN, A. S. y TINETTI, M. E. (2016). «Precision medicine to precision care: managing multimorbidity». The Lancet, 388 (10061), 2721-2723. 
[12] BIRD, R. M. (1971). «Wagner's o Law'of Expanding State Activity». Public Finance, Finances publiques, 26 (1), 1-26.

[13] BLAY, C. (2012). Programa de prevención y atención a la cronicidad (PPAC). Departamento de Salud de Cataluña.

[14] BOLETÍN OFICIAL DEL ESTADO (2011). Real Decreto 174/2011, de 11 de febrero, por el que se aprueba el baremo de valoración de la situación de dependencia establecido por la Ley 39/2006, de 14 de diciembre, de Promoción de la Autonomía Personal y Atención a las personas en situación de dependencia.

[15] CASTELLS-OLIVERES, A. M. y BOSCH-ROCA, N. (1998). El futuro del Estado del Bienestar: algunas líneas de reflexión. Civitas.

[16] COMISIÓN EUROPEA (2015). «The 2015 Ageing Report: Economic and budgetary projections for the 28 EU Member States (2013-2060)». Directorate-General for Economic and Financial Affairs, Economic Policy Committee (AWG), 3.

[17] CONDE-RUIZ, J. I. y OCAÑA-ORBIS, C. (2007). «Análisis cuantitativo del estado de bienestar en Europa: Modelos y resultados». Documento de Trabajo FEDEA, 3, 03.

[18] CONSEJERÍA DE SALUD DE ANDALUCÍA (2012). Plan andaluz de atención integrada a pacientes con enfermedades crónicas. 2012-2016.

[19] CONSEJERÍA DE SANIDAD DE CASTILLA Y LEÓN (2013). Estrategia de atención al paciente crónico en Castilla y León.

[20] CONSEJERÍA DE SANIDAD DE LA COMUNIDAD DE MADRID (2013). Estrategia de atención a pacientes con enfermedades crónicas en la Comunidad de Madrid.

[21] CORRAL-GUDINO, L.; CENGOTITA-BENGOA, M. B.; JORGE-SÁNCHEZ, R. y GARCÍA-APARICIO, J. (2017). «El paciente y su travesía entre la atención primaria y la hospitalaria. Revisión sistemática de ensayos clínicos para la implementación de herramientas para la integración en España». Anales del Sistema Sanitario de Navarra, 40 (3), 443-459.

[22] COSTA-FONT, J.; JIMÉNEZ-MARTÍNEZ, S. y VILAPLANA, C. (2016). «Does long-term care subsidisation reduce hospital admissions?». CESifo Working Papers, 6078.

[23] CRONIN, J.; MURPHY, A. y SAVAGE, E. (2017). «Can chronic disease be managed through integrated care cost-effectively? Evidence from a systematic review». Irish Journal of Medical Science, 186, 827.

[24] DEL POZO-RUBIO, R.; PARDO-GARCÍA, I. y ESCRIBANO-SOTOS, F. (2017). «El copago de dependencia en España a partir de la reforma estructural de 2012». Gaceta Sanitaria, 31 (1), 23-29.

[25] DEPARTAMENTO DE SANIDAD Y CONSUMO DE PAÍS VASCO (2010). Estrategia para afrontar el reto de la cronicidad en Euskadi.

[26] DIRECCIÓ GENERAL DE REGULACIÓ, PLANIFICACIÓ I RECURSOS SANITARIS DE LA GENERALITAT DE CATALUNYA (2012). Programa de prevenció $i$ atenció a la cronicitat. Document conceptual.

[27] DESMEDT, M.; VERTRIES S.; HELLINGS, J.; BERGS, J.; DESSERS, E.; VANFRUNKELSVEN, P.; VRIJHOEF, H.; ANNEMANS, L.; VERHAEGHE, N. y PETROVIC, M. (2016). «Economic Impact of Integrated Care Models for Patients with Chronic Diseases: A Systematic Review». Value in Health, 19 (6), 892-902.

[28] ESPING-ANDERSEN, G. (1990). The Three Worlds of Welfare Capitalism. Princeton, NJ, Princeton University Press. 
[29] ESPING-ANDERSEN, G., y PALIER, B. (2010). Los tres grandes retos del Estado del bienestar. Grupo Planeta.

[30] EUROSTAT (2018). STATISTICS-Explained. Government expenditure by function.

[31] FUSI, J. P. y PALAFOX, J. (1997). España: 1808-1996. El desafí de la modernidad. Madrid, Espasa.

[32] GARCÍA-CALVENTE, M. M.; MATEO-RODRÍGUEZ, I. y EGUIGUREN, A. P. (2004). «El sistema informal de cuidados en clave de desigualdad». Gaceta Sanitaria, $18(4), 132-139$.

[33] GONZÁLEZ-PÁRAMO, J. y RAYMOND BARA, J. (1988). «¿Por qué el gasto público tiende a crecer?». Papeles de Economía Española, 37, 145-149.

[34] IMSERSO (2018). «Datos sobre el estado de situación de la gestión del Sistema para la Autonomía y Atención a la Dependencia (SAAD)». Disponible en http://www.imsersodependencia.csic.es/estadisticas/saad/index.html.

[35] INSALUD-IMSERSO (1995). Jornadas europeas sobre atención a las personas mayores dependientes. La respuesta socio-sanitaria. Madrid.

[36] INSTITUTO NACIONAL DE ESTADÍSTICA (2018). Demografía y sociedad. Estadísticas del censo continuo.

[37] INSTITUTO NACIONAL DE ESTADÍSTICA (2008). Encuesta sobre discapacidades, autonomía personal y situaciones de dependencia.

[38] JAÉN-GARCÍA, M. (2004). La ley de Wagner: un análisis sintético. Madrid, Instituto de Estudios Fiscales.

[39] JIMÉNEZ-MARTÍN, S.; OLIVA, J.; VILAPLANA PRIETO, C.; HERRERA, E.; MUÑOZ, I. y MARTÍN, V. (2011). Sanidad y dependencia; matrimonio o divorcio. Madrid, Círculo de la Sanidad.

[40] KODNER, D. L. y KYRIACOU, C. K. (2000). «Fully integrated care for frail elderly: two American models». International Journal of Integrated Care, 1.

[41] LÓPEZ-CASANOVAS, G. y DEL POZO RUBIO, R. (2010). «La protección social de los problemas de dependencia en España». Documentos de Trabajo Fundación CASER-CRES, 1.

[42] LÓPEZ CASASNOVAS, G. (2009). «Análisis económico de la cobertura de la dependencia: algunas reflexiones sobre las causas y consecuencias de los déficits de la regulación actual». Cuadernos Aragoneses de Economía, 19 (2), 245-263.

[43] LUHMANN, N. y VALLESPÍN, F. (1993). Teoría política en el Estado de Bienestar: Madrid, Alianza.

[44] MESTHENEOS, E. y TRIANTAFILLOU, J. (2005). Supporting family carers of older people in Europe-The Pan-European background report. Siglo del Hombre Editores.

[45] MINISTERIO DE SANIDAD. (2018). El Sistema Público de Servicios Sociales. Plan Concertado de Prestaciones Básicas de Servicios Sociales en Corporaciones Locales 2015-2016. Madrid.

[46] MINISTERIO DE SANIDAD, SERVICIOS SOCIALES E IGUALDAD (2012). Estrategia para el abordaje de la cronicidad en el Sistema Nacional de Salud. Madrid.

[47] NEW HEALTH FOUNDATION (2017). Experiencias de atención integrada en España. Informe OMIS 2016. Observatorio de Modelos Integrados en Salud.

[48] MUIR, T. (2017). «Measuring social protection for long-term care». OECD Health Working Papers, 93.

[49] MUSGRAVE, R. A. (1969). Fiscal Systems. New Haven and London: Yale University Press. 
[50] NAVARRO, V. (2000). Globalización económica, poder político y Estado del bienestar: Ariel.

[51] NOLTE, E. y PITCHFORTH, E. (2014). What is the evidence on the economic impacts of integrated care? . World Health Organization Regional Office for Europe and European Observatory on Health Systems and Policies. Policy Summary 11.

[52] NUÑO-SOLINÍS, R. (2016). «Desarrollo e implementación de la estrategia de cronicidad del País Vasco: lecciones aprendidas». Gaceta Sanitaria, 30, 106-110.

[53] OCDE (2017). Health at Glance.

[54] OLIVA, J. (2014). «Análisis y líneas de avance en tres dimensiones del sistema de autonomía personal y atención a la dependencia». Revista de Actas de la Dependencia Fundación Caser, 12.

[55] OLIVA, J.; VILAPLANA, C. y OSUNA, R. (2011). «El valor social de los cuidados informales provistos a personas mayores en situación de dependencia en España». Gaceta Sanitaria, 25, 108-114.

[56] ORGANIZACIÓN MUNDIAL DE LA SALUD (2015). World report on ageing and health. Geneva, World Health Organization.

[57] ORGANIZACIÓN MUNDIAL DE LA SALUD (2016). World Health Statistics 2016: Monitoring Health for the SDGs Sustainable Development Goals: Geneva, World Health Organization.

[58] ORTEGA-ORTEGA, M.; MONTERO-GRANADOS, R. y JIMÉNEZ-AGUILERA, J. D. (2018). «Differences in the economic valuation and determining factors of informal care over time: the case of blood cancer». Gaceta Sanitaria, 32 (5), 411-417.

[59] OSAKIDETZA (2010). Estrategia para afrontar el reto de la cronicidad en Euskadi. Eusko Jaurlaritza. Gobierno Vasco. Departamento de Sanidad y Consumo.

[60] PEÑA-LONGOBARDO, L. M. y OLIVA-MORENO, J. (2015). «Economic valuation and determinants of informal care to people with Alzheimer's disease». The European Journal of Health Economics, 16 (5), 507-515.

[61] PÉREZ-GARCÍA, F. (2011). Crecimiento y competitividad: trayectoria y perspectivas de la economía española. Madrid, Fundacion BBVA.

[62] PINES, J.; SELEVAN, J.; MCSTAY, F.; GEORGE, M. y MCCLELLAN, M. (2015). Kaiser Permanente-California: a model for integrated care for the ill and injured. The Brookings Institution.

[63] SOSVILLA-RIVERO, S. y MORAL-ARCE, I. (2011). «Estimación de los beneficiarios de prestaciones de dependencia en España y del gasto asociado a su atención para 20072045». Gaceta Sanitaria, 25, 66-77.

[64] TEDD DE LORCA, P. (1996). «De la primera a la segunda Restauración. El Sector Público y la modernización de la economía española, 1875-1975». Ayer (21), 15-49.

[65] TRIBUNAL DE CUENTAS. (2013). Informe de fiscalización 977 de la gestión económico-financiera y de la aplicación de la Ley 39/2006, de 14 de diciembre, de promoción de la Autonomía Personal y Atención a las personas en situación de dependencia. Madrid, Tribunal de Cuentas.

[66] URTARAN-LARESGOITI, M.; ÁLVAREZ-ROSETE, A. y NUÑO-SOLINÍS, R. (2018). «A system-wide transformation towards integrated care in the Basque Country: A realist evaluation». International Journal of Health Coordination, 21 (3), 98-0108. 